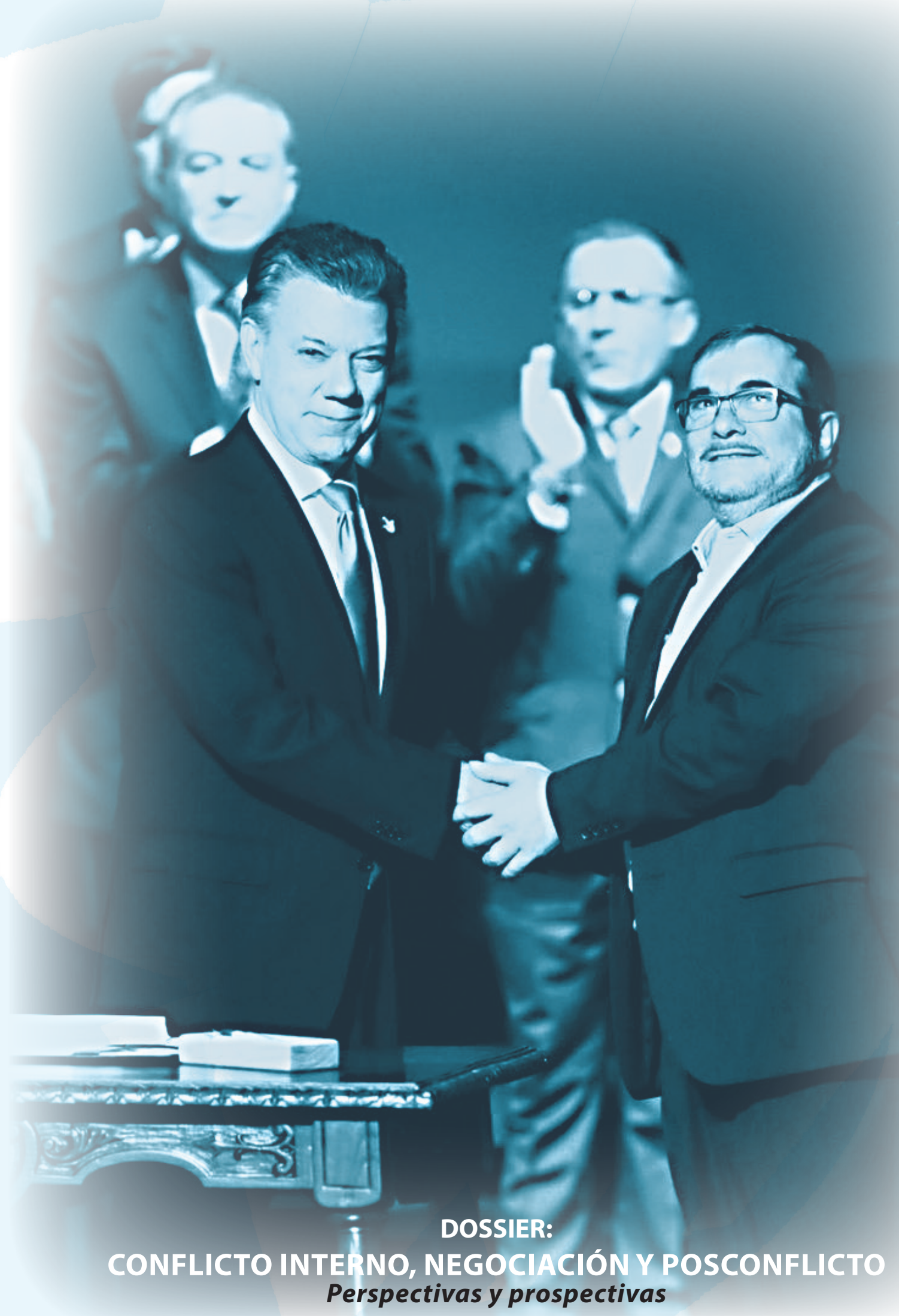




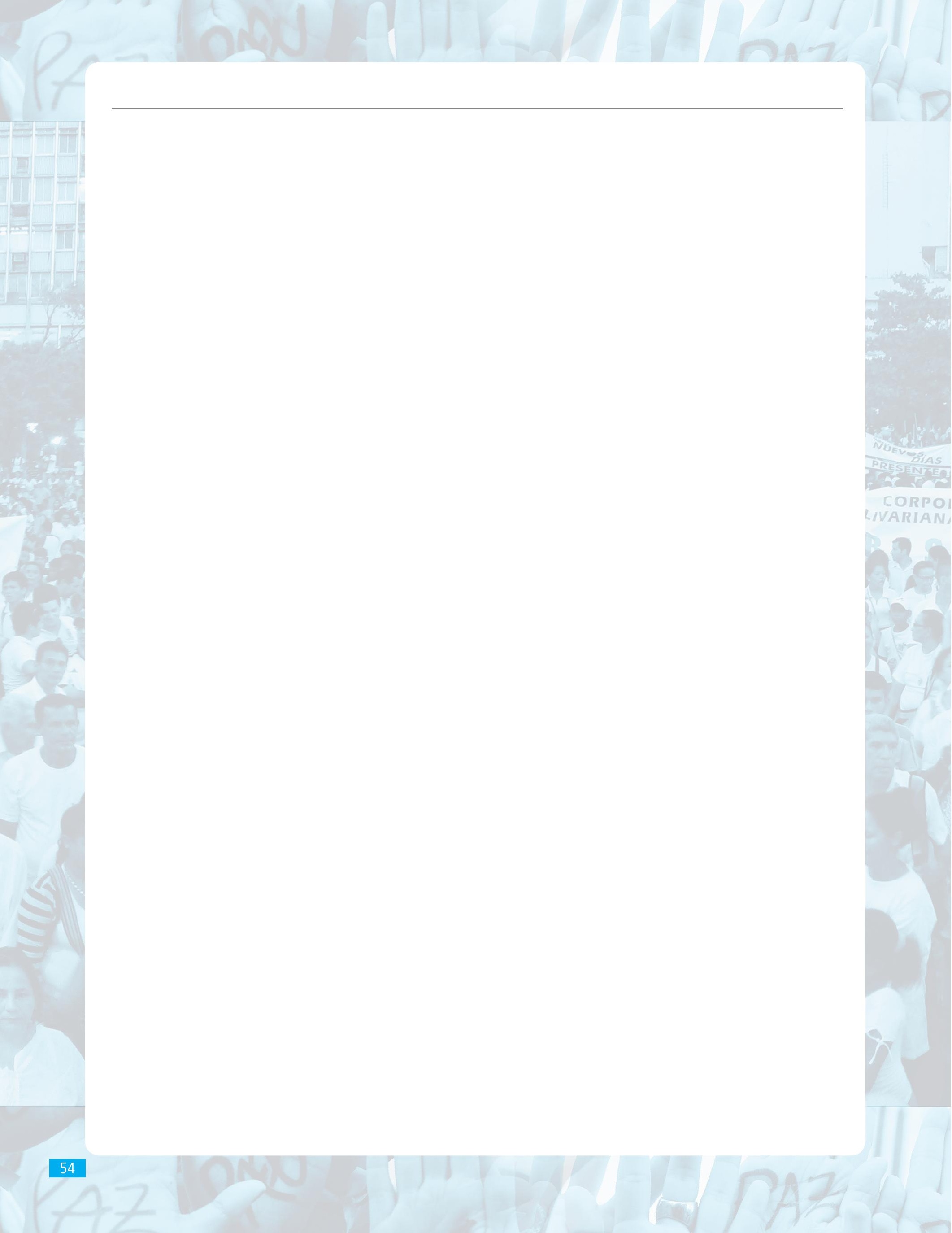




\title{
Postconflicto en Colombia
}

\section{Post-conflict in Colombia}

\author{
Luis Fernando Gamboa Fandiño ${ }^{1}$ \\ Jhoan Sebastian Londoño Piedrabita
}

Universidad del Valle

\section{Resumen}

Es importante establecer los parámetros en los que el posconflicto se va a desarrollar, ya que es de suma relevancia analizar todos los aspectos que se deben tener en cuenta después de un eventual tratado en el proceso de paz, en el que todos los actores involucrados en este conflicto, que abarca tanto a las víctimas como a los victimarios, deben responsabilizarse y asumir su respectivo rol en búsqueda de acuerdos para la reparación integral de las víctimas, a quienes se les debe reconocer sus derechos vulnerados, además de apoyar a las comunidades desplazadas, las principales damnificadas en este conflicto, para su retorno a las zonas rurales. Por otra parte, los victimarios deben comprometerse con la reinserción a la sociedad, paralela y simultáneamente con los procesos sociales de perdón, reconciliación y reparación de las víctimas, para evitar el resurgimiento de otros grupos armados como en su momento ocurrió con las AUC (Autodefensas Unidas de Colombia). Se plantean dos preguntas clave: la primera ¿̇i es posible una reinserción exitosa a la sociedad? Ya que se debe tener en cuenta la ideología de los diferentes individuos y como ha sido su socialización tanto primaria como secundaria frente a las circunstancias que lo llevaron a formar parte de estos grupos armados; y en segundo lugar entender ¿cómo afectan o contribuyen estas ideologías a las generaciones colombianas futuras? para que eventualmente no surjan más grupos armados en el país.

Palabras clave: autodefensas, diálogos de paz, Fuerzas Armadas Revolucionarias de Colombia (FARC), guerrillas.

\section{Abstract}

It is important to establish the parameters within which the post-conflict will develop, as it is important to analyze all the aspects to be taken into account after a treaty eventual peace process, in which all stakeholders in this conflict that covers both the victims and the perpetrators, must take responsibility and assume their respective role in seeking agreements for the reparation of victims where they should recognize their rights which were violated and support to displaced communities for return to rural areas which have been the main affected in this conflict; Moreover offenders must commit to the reintegration into society, parallel and simultaneously with the social processes of forgiveness, reconciliation and reparation for victims to avoid precisely the resurgence of other armed groups as at the time it was with the AUC (united self-defense of Colombia). Two key questions, the first describes what if successful reintegration into society is possible? Since it must take into account the ideology

1 Luis Fernando Gamboa Fandiño: estudiante de Contaduría Pública - Universidad del Valle, sede Palmira. Correo: luis. gamboa@correounivalle.edu.co

2 Jhoan Sebastian Londońo Piedrahita: estudiante de Contaduría Pública - Universidad del Valle, sede Palmira. Integrante del Grupode Investigación Contable de la Universidad del Valle. Correo: joan.londono@correounivalle.edu.co 
of various individuals and as has been his primary socialization as both secondary to the circumstances that led him to join these armed groups; and secondly to understand how these ideologies affect or contribute to future Colombian generations? To not more armed groups eventually arise in the country.

Keywords: united self-defense of Colombia, partisan warfare, peace talks, Revolutionary Armed Forces of Colombia (FARC).

Recibido: 22 de septiembre de 2015

Aceptado: 01 de noviembre de 2015

\section{Introducción}

Según la Universidad del Rosario se describe post-conflicto como: el periodo de tiempo que sigue a la superación total o parcial de los conflictos armados. Puede entenderse como un concepto de un único atributo que es la reducción del número de homicidios relacionados con el conflicto por debajo de cierto umbral determinado, que le otorga o le niega el estatus de conflicto armado activo; de acuerdo al paradigma social del posconflicto en Colombia, lo que se esperaría posteriormente al tratado de paz es, en primer lugar la búsqueda de un nuevo rol a ocupar para los desmovilizados de los diferentes bloques de las Fuerzas Armadas Revolucionarias de Colombia - Ejército del Pueblo (FARC-EP) que decidan acogerse bajo los beneficios que brinda el Estado, ya que son precisamente los individuos que hacen parte de estas guerrillas los que después de un eventual tratado en el proceso de paz deben resocializarse, lo que implicaría cambiar toda la estructura mental que previamente habían adquirido, ya sea por voluntad propia en dicho momento o por presión de los jefes guerrilleros.

La pregunta que se debe analizar dado el contexto del pos-conflicto es ¿si posible una reinserción exitosa a la sociedad de los futuros desmovilizados?; observando la realidad tanto objetiva como subjetiva, dada la idiosincrasia, mentalidad e ideología del individuo reinsertado a la sociedad, de estas estructuras de las FARC-EP, deben de tener un cambio drástico, ya que no se trata única y explícitamente de los jerarcas de las guerrillas sino también de todos los individuos que, aunque con menos poderío, cumplen una función interna dentro de las mismas organizaciones rebeldes, muchos de ellos con ideologías marxistas y leninistas, entonces ¿̨hasta dónde se podrá transformar esta ideología para que eventualmente no surjan más grupos armados en Colombia? Y, aún más complejo que este planteamiento, es entender ¿¿cómo afectan o contribuyen estas ideologías a las generaciones colombianas futuras? para que así suceda una transformación en la ideología de los ciudadanos que permita brindar a las nuevas y futuras generaciones, desde el comienzo, es decir desde la socialización primaria, unas herramientas cognitivas correctas, responsables frente a los valores y virtudes que como nación debemos, y estamos en la obligación de pregonar.

\section{Una mirada crítica a la historia}

En las últimas décadas se vislumbra un "odio" generalizado que se ve reflejado en muchos sectores de la sociedad, principalmente los sectores populares, que no cuentan con las posibilidades económicas para tener una buena calidad de vida, lo que genera en ellos un resentimiento que se ve reflejado en la violencia que se presenta en la vida cotidiana en el país; pero que no termina únicamente en delincuencia común sino que también este descontento se ha convertido en una ideología de izquierda que desde hace más de cincuenta ańos azota al país y lo mantiene en un clima de constante zozobra y terror frente a lo que estos grupos armados de extrema-izquierda originan, ya que en vez de buscar que su ideología sea reconocida, validada y apoyada por las distintas comunidades de Colombia y el mundo, por medio de un ámbito político que les permita de manera sana dar a conocer sus propuestas, 
lo hacen mediante la intimidación y el terrorismo; según Peter Berger y Thomas Luckmann, 2001., cuando ocurre una internalización, "yo no solo comprendo los procesos subjetivos momentáneos del otro: comprendo el mundo en que él vive, y ese mundo se vuelve mío" Pág. 165; esto se vé reflejado claramente en cómo fue el nacimiento de las primeras guerrillas colombianas.

Según Erich Saumeth Cadavid, consultor e investigador en asuntos de defensa, seguridad ciudadana y orden público, en su artículo "Historia de la Guerrilla en Colombia", las primeras guerrillas de Colombia nacen por la necesidad de defender, y es lógico ya que con el Frente Nacional, el cual limitó la participación de otros partidos políticos y no tuvo en cuenta los ideales del pueblo, por ende las guerrillas buscaban defender dichos ideales, no obstante, al no tener la participación política decidieron emprender un camino sangriento en contra del Estado y del mismo pueblo, que en la actualidad les ha costado la legitimización del mismo.

En el artículo "Guerra y política en la sociedad colombiana", el autor Gonzalo Sánchez Gómez, presenta de manera cronológica y real los diferentes conflictos que han ido apareciendo en Colombia desde su creación como república independiente, resaltando conflictos de acuerdo con la época y la necesidad social predominante en ciertos momentos de la historia.

En primer lugar, analiza las diferentes guerras civiles que han ido ocurriendo a lo largo de la historia en diferentes naciones y que son de similar índole; primeramente la centralización y el orden político departamental, el equilibrio de poderes sustentado desde diferentes puntos de vista en Colombia, tanto por el partido conservador como por el partido liberal, los cuales se reconocen como los dos partidos históricos y con mayor recorrido político en Colombia; en segundo lugar aparece la definición "modelos de desarrollo" en donde se analizaría la competitividad a nivel mundial, la repartición de trabajo mundial en donde Colombia, según el acuerdo, se compromete a enviar materias primas, este último planteamiento ha sido altamente debatido por distintos sectores económicos del país, entre ellos las corrientes de pensamiento de izquierda los cuales condenan vehementemente por su naturaleza de enajenación de los recursos naturales, su explotación en extremo y la poca igualdad con la destinación de los recursos producto de esas negociaciones entre el Estado colombiano y naciones extranjeras, como por ejemplo, Estados Unidos.

Desde la creación de las primeras guerrillas en Colombia, producto de la violencia política, nace la necesidad de crear un grupo armado con fuerza militar suficiente para hacerle frente al legitimo Estado, que en este caso estaría representado por el gobierno de turno, básicamente para combatirlo y posteriormente ocupar su lugar por medio de un golpe de Estado; por medio de estas acciones revolucionarias desde sus comienzos, los grupos guerrilleros han querido generar un cambio en las corrientes de pensamiento predominantes del país, llevando a un punto medio el equilibrio social, representando a las clases menos favorecidas del país, las clases populares, a quienes se les ultrajan sus derechos, como lo son los campesinos y las familias que hacen parte de las clases más bajas del país; tampoco se puede negar que el nacimiento de la guerrilla colombiana se ve fuertemente influenciada por la revolución cubana, el golpe de Estado al régimen de Batista y la nueva corriente de pensamiento comunista que iba creciendo a lo largo de Latinoamérica.

En un comienzo es clara la ideología guerrillera de los diferentes grupos armados que surgen en Colombia, los cuales podríamos denominar de primera generación, como es el caso del ELN y el EPL, con una ideología marxista, de igualdad y lucha por una distribución de las riquezas más equitativa; su discurso se encamina hacia la igualdad de clases, similar al punto de vista de Slavoj Zizek en la hegemonía y sus síntomas, donde se puede observar una óptica pragmática por parte del autor, que analiza el contexto mundial y más específicamente soviético, dado el gran poder del discurso y, más allá de ello, la "legibilidad" con la que cierto grupo político consigue una identificación por parte 
de la comunidad; pero este punto de vista pragmático no solo se debe ver como una "clarificación semántica" sino una universalidad de la noción.

Desde sus comienzos, la guerrilla siempre ha buscado desplazarse a lo largo y ancho del territorio nacional, además de buscar siempre acrecentar el número de combatientes de sus filas y esto último lo ha hecho en contra de los derechos humanos, al obligar a menores de edad a hacer parte de sus filas en contra de su voluntad, lo que resulta contradictorio con la ideología de justicia que desean pregonar; también al analizar la economía de las guerrillas y como consiguen entradas de dinero para subsistir, aprovisionarse de armas y mantenerse liquidas, financieramente hablando, se observa la contradicción de la lucha por los ideales que supuestamente representan ya que su financiación se presenta por medio de actividades económicas que además de ilegales generan mucho daño a la sociedad y al país; el secuestro, la extorsión a ganaderos y comerciantes en diferentes regiones del país que tanto daño le hace a la economía de la nación; la explotación de minería ilegal y destrucción de oleoductos que tanto daño le hace al medio ambiente cuando este petróleo es derramado en el mar; y principalmente el narcotráfico que por medio de diferentes bloques guerrilleros delictivos que operan en el país hoy en día mantienen en pie dicho negocio, generando extraordinarias entradas de dinero, convirtiendo a la FARC y ELN como unas de las guerrillas con mayor recursos económicos del mundo.

\section{Conclusiónes}

Una reinserción en la vida civil por parte de los combatientes de las FARC traza nuevos desafíos tanto para ellos como para el Estado y la sociedad. Colombia sabe que es firmar tratados de paz, en efecto, se logra evidenciar los acuerdos alcanzados con el movimiento Guerrillero M-19 en 1990 y con el grupo paramilitar Autodefensas Unidas de Colombia (AUC) en 2006, siendo esté el más reciente. Por estas razones el país no es ajeno a dicha realidad.

En 2003 el Estado colombiano creó el Programa para la Reincorporación a la Vida Civil (PRVC) del Ministerio del Interior y de Justicia, que en el año 2011 paso a ser la Agencia Colombiana para la Reintegración (ACR), encargada de conducir a los desmovilizados a superar la condición de vulnerabilidad y lograr con ello la correcta inserción en la sociedad. Es de ahí que 9.038 desmovilizados hayan culminado su proceso exitosamente y un poco menos de la mitad se encuentran adelantando su inclusión. Según estadísticas de este ente, el $76 \%$ de los vinculados se encuentran en la legalidad y $72,3 \%$ se encuentran ocupados en alguna actividad (15.459). No obstante, por más que el Estado brinde el acompańamiento en las dimensiones laborales, educativa, salud, seguridad entre otros, el éxito que ha tenido el programa no es suficiente.

En la sociedad colombiana existen temores en un porcentaje de colombianos, desconfianzas, dudas y/o sospechas que abruman a los ciudadanos e impiden reconocer y acoger como parte de la sociedad a los desmovilizados, es así como lo expresa el profesor William Fernando Torres. Y como no temer si muchos de los desmovilizados vuelven a delinquir y así lo deja saber el Alto Comisionado de las Naciones Unidas para los derechos humanos en Colombia: "Los grupos post-desmovilización son el reto principal en seguridad pública hoy en día en Colombia y ellos siguen siendo violadores de derechos humanos". Además, sólo por citar un ejemplo de los cientos que pueden haber en nuestro país, se puede hablar del secuestro de una niña en Cali a manos de desmovilizados de las FARC, quienes vivían con la familia de la niña y al darse cuenta que tenían un CDT por cincuenta millones de pesos procedieron a secuestrarla con la intención de cobrar el rescate y luego asesinarla; uno de ellos, Alias "Tumaco" estuvo vinculado al programa del ACR y salió por voluntad propia del mismo. 
Para concluir se puede afirmar que cada proceso de desmovilización es diferente, y el país sabe eso. El ACR y el gobierno han venido adelantando estrategias para una eventual desmovilización, pero no adelantan al país para entender y aceptar dicha desmovilización (si llegase a ocurrir). Y es allí donde William Torres hace la pregunta sobre: ¿Educar para la inserción social o reconstruir lo colectivo en colectivo?. Colombia aún no se está preparando para el post-conflicto.

\section{Referencias}

Ugarriza, Juan E. 2013. "La dimensión política del postconflicto. Avances conceptuales y empiricos", Colombia Internacional 77.

Berger, Peter y Luckmann, Thomas (2001). La sociedad como realidad subjetiva, internalización de la realidad-Socialización primaria.

Saumeth Cadavid Erich. (2010). Historia de la Guerrilla en Colombia. 2015, de Universidade Federal de Juiz de Fora. Recuperado de: http://ecsbdefesa.com.br/defesa/fts/HGC.pd

Boulding KE. Conflict and defense. New York: Harper and Raw Publishers; 1962.

Rettberg A, Camacho A, Chaux E, García A, Iturralde M, Sánchez F, Sanz de Santamaría A, Wills L. Preparar el futuro: conflicto y post-conflicto en Colombia. Bogotá:

Gonzalo Sánchez Gómez. (1990). "Guerra Y Politica en la sociedad Colombiana”. Bogotá: Revista Análisis Político.

Slavoj Zizek. (2008). "En defensa de la intolerancia". -: Sequitur. 